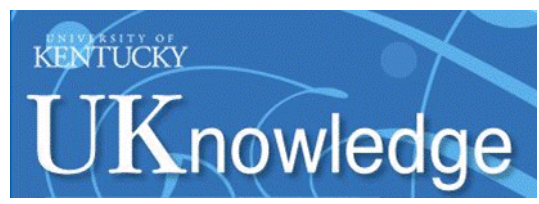

University of Kentucky

UKnowledge

$12-7-2021$

\title{
Treatment of Full-Thickness Acetabular Chondral Flaps during Hip Arthroscopy: Bone Marrow Aspirate Concentrate versus Microfracture
}

\author{
Michael P. Kucharik \\ Massachusetts General Hospital \\ Paul F. Abraham \\ University of Southern California \\ Mark R. Nazal \\ University of Kentucky, mark.nazal@uky.edu \\ Nathan H. Varady \\ Hospital for Special Surgery \\ Christopher T. Eberlin \\ Massachusetts General Hospital \\ Follow this and additional works at: https://uknowledge.uky.edu/orthopaedicsurgery_gradpub \\ I next page for additional authors \\ Part of the Sports Medicine Commons \\ Right click to open a feedback form in a new tab to let us know how this document benefits you.
}

\section{Repository Citation}

Kucharik, Michael P.; Abraham, Paul F.; Nazal, Mark R.; Varady, Nathan H.; Eberlin, Christopher T.; Meek, Wendy M.; Naessig, Sara A.; and Martin, Scott D., "Treatment of Full-Thickness Acetabular Chondral Flaps during Hip Arthroscopy: Bone Marrow Aspirate Concentrate versus Microfracture" (2021). Orthopaedic Surgery and Sports Medicine Resident Research. 1.

https://uknowledge.uky.edu/orthopaedicsurgery_gradpub/1

This Article is brought to you for free and open access by the Orthopaedic Surgery and Sports Medicine at UKnowledge. It has been accepted for inclusion in Orthopaedic Surgery and Sports Medicine Resident Research by an authorized administrator of UKnowledge. For more information, please contact UKnowledge@lsv.uky.edu. 


\title{
Treatment of Full-Thickness Acetabular Chondral Flaps during Hip Arthroscopy: Bone Marrow Aspirate Concentrate versus Microfracture
}

\author{
Digital Object Identifier (DOI) \\ https://doi.org/10.1177/23259671211059170 \\ Notes/Citation Information \\ Published in Orthopaedic Journal of Sports Medicine, v. 9, issue 12, 23259671211059170. \\ (C) The Author(s) 2021
}

This article is distributed under the terms of the Creative Commons Attribution-NonCommercial-NoDerivs 4.0 License (https://creativecommons.org/licenses/by-nc-nd/4.0/) which permits non-commercial use, reproduction and distribution of the work as published without adaptation or alteration, without further permission provided the original work is attributed as specified on the SAGE and Open Access pages (https://us.sagepub.com/en-us/nam/open-access-at-sage).

Authors

Michael P. Kucharik, Paul F. Abraham, Mark R. Nazal, Nathan H. Varady, Christopher T. Eberlin, Wendy M. Meek, Sara A. Naessig, and Scott D. Martin 


\title{
Treatment of Full-Thickness Acetabular Chondral Flaps During Hip Arthroscopy
}

\section{Bone Marrow Aspirate Concentrate Versus Microfracture}

\author{
Michael P. Kucharik, ${ }^{*,}$ BS, Paul F. Abraham, ${ }^{\ddagger}$ MD, Mark R. Nazal, ${ }^{\S}$ MD, MPH, \\ Nathan H. Varady," MD, MBA, Christopher T. Eberlin, ${ }^{\dagger}$ BS, Wendy M. Meek, ${ }^{\dagger}$ BBA, \\ Sara A. Naessig, ${ }^{\dagger}$ BS, and Scott D. Martin, ${ }^{\dagger}$ MD \\ Investigation performed at Sports Medicine, Department of Orthopaedic Surgery, Massachusetts \\ General Hospital, Mass General Brigham, Boston, Massachusetts, USA
}

Background: The optimal treatment strategy for patients with full-thickness chondral flaps undergoing hip arthroscopy is controversial.

Purpose: To compare functional outcomes of patients who underwent bone marrow aspirate concentrate (BMAC) application with those of patients who underwent microfracture.

Study Design: Cohort study; Level of evidence, 3.

Methods: This was a retrospective case series of prospectively collected data on patients who underwent arthroscopic acetabular labral repair by 1 surgeon between June 2014 and April 2020. The inclusion criteria for this study were age $\geq 18$ years, preoperative radiographs of the pelvis, arthroscopic acetabular labral repair, exposed subchondral bone with overlying chondral flap seen at the time of hip arthroscopy, microfracture or BMAC to address this lesion, and completed patient-reported outcome measures (PROMs) (International Hip Outcome Tool-33 [iHOT-33], Hip Outcome Score-Activities of Daily Living [HOS-ADL], Hip Outcome Score-Sports Subscale [HOS-Sport], modified Harris Hip Score [mHHS], and visual analog scale [VAS] for pain) at enrollment and 12-month follow-up. Clinical outcomes were assessed using PROM scores.

Results: A total of 81 hips with full-thickness chondral flaps were included in this study: 50 treated with BMAC and 31 treated with microfracture. There were no significant differences between groups in age, sex, body mass index, tear size, radiographic osteoarthritis, or radiographic femoroacetabular impingement. In the BMAC cohort, all PROM scores improved significantly from preoperatively to follow-up: 41.7 to 75.6 for iHOT-33, 67.6 to 91.0 for HOS-ADL, 41.5 to 72.3 for HOS-Sport, 59.4 to 87.2 for mHHS, and 6.2 to 2.2 for VAS pain $(P<.001$ for all). In the microfracture cohort, the score improvements were 48.0 to 65.1 for iHOT-33 $(P=.001), 80.5$ to 83.3 for HOS-ADL $(P=.275), 59.2$ to 62.4 for HOS-Sport $(P=.568), 70.4$ to 78.3 for $\mathrm{mHHS}(P=.028)$, and 4.9 to 3.6 for VAS pain $(P=.036)$. Regarding clinically meaningful outcomes, $77.6 \%$ of the BMAC group and $50.0 \%$ of the microfracture group met the minimal clinically important difference for iHOT-33 at the 12-month follow-up $(P=.013)$.

Conclusion: Patients with full-thickness chondral flaps at the time of hip arthroscopy experienced greater improvements in functional outcome scores at the 12-month follow-up when treated with BMAC as opposed to microfracture.

Keywords: hip arthroscopy; acetabular labral repair; chondral flap; bone marrow aspirate concentrate; microfracture

Femoroacetabular impingement (FAI) has been associated with hip pain, acetabular labral injuries, and chondral lesions of the femoral head and acetabulum. ${ }^{2,31}$ In patients with combined FAI (concomitant cam and pincer lesions), shearing forces during flexion and internal rotation cause an asymmetric femoral head to impinge against

The Orthopaedic Journal of Sports Medicine, 9(12), 23259671211059170 DOI: 10.1177/23259671211059170

(C) The Author(s) 2021 an overcovered acetabular rim. ${ }^{21}$ Initially, acetabular cartilage remains macroscopically intact, but it can eventually become delaminated, fissured, and cracked-a manifestation that has been reported in $44 \%$ to $86 \%$ of patients with FAI undergoing hip arthroscopy. ${ }^{3,13,22,30,35,37}$ In some cases, the acetabular cartilage may completely rupture from underlying subchondral bone starting in the periphery of the joint and progressing centrally to form a full-thickness, "outside-in" chondral flap. ${ }^{21,23}$ While severe, widespread cartilage delamination has been associated with poor clinical outcomes after hip arthroscopy, ${ }^{18}$ the optimal treatment

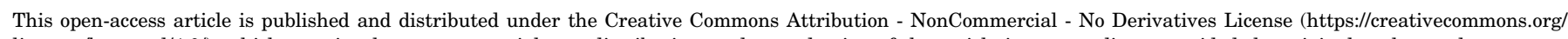

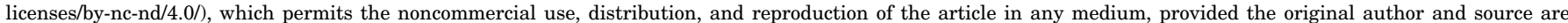

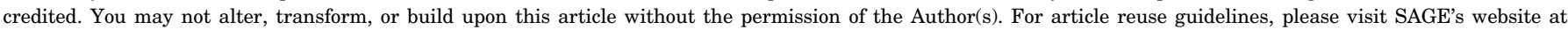
http://www.sagepub.com/journals-permissions. 
strategy for patients with isolated, full-thickness chondral flaps is controversial. ${ }^{12,19,26,29,36,38,42}$

Currently, few clinical studies have compared functional outcomes for different strategies for the treatment of fullthickness chondral flaps. Some studies have suggested that chondral flaps serve as a source of impingement and their removal by excision, debridement, or abrasion would allow the lesion to heal itself over time. ${ }^{4,8,10}$ More recent studies have advised the fixation of the flap using fibrin adhesive, autologous scaffolding material, implanted collagen membrane, or underlying microfracture. ${ }^{12,20,26,38,42}$ These preservation techniques are theoretically ideal since chondral flaps have been reported to contain up to $90 \%$ viable chondrocytes. ${ }^{29,43}$ However, the extracellular matrix (ECM) surrounding the viable chondrocytes is largely disturbed, which warrants the use of a substance to bridge the chondral flap to subchondral bone to allow for long-term attachment and distribution of new ECM. ${ }^{24,43}$

While microfracture has shown favorable results in treating patients with full-thickness chondral damage, ${ }^{11,25}$ some studies have suggested downsides, such as a weakened trabecular bone structure leading to an increased risk of conversion to total hip arthroplasty (THA) with extended follow-up. ${ }^{14,28}$ Accounting for these concerns, surgeons could use bone marrow aspirate concentrate (BMAC) as a method to bridge the chondral flap to subchondral bone without violating the underlying trabecular bone. BMAC has shown promising results for the treatment of other chondral insults, and its utilization during hip arthroscopy could potentially allow for long-standing reattachment of biologically viable chondral flaps. ${ }^{1,5,7,40}$

The current study aimed to add to the growing body of literature addressing strategies for the preservation of full-thickness chondral flaps during hip arthroscopy by reporting the utilization of BMAC as a unique technique for fixation and comparing these results to those of patients who underwent microfracture. We hypothesized that patients with full-thickness chondral flaps treated with BMAC application would report superior functional outcomes compared with similar patients treated with microfracture.

\section{METHODS}

\section{Study Population and Design}

This study was approved by our institutional review board and patients provided informed consent. Data for this study were prospectively collected and retrospectively reviewed. All included patients underwent arthroscopic acetabular labral repair by the senior surgeon (S.D.M.) between June 2014 and April 2020 and completed patient-reported outcome measures (PROMs) at enrollment and 12-month follow-up. Inclusion criteria were age $\geq 18$ years; preoperative radiographs of the pelvis; arthroscopic acetabular labral repair; and exposed subchondral bone with overlying chondral flap seen at the time of hip arthroscopy, with microfracture or BMAC to address this lesion. Exclusion criteria were previous hip arthroscopy and arthroscopic acetabular labral debridement.

All patients initially evaluated with hip pain at the senior author's (S.D.M.) clinic received hip and pelvis radiographs and a thorough physical examination, including provocation testing of the labrum and evaluation for FAI syndrome. ${ }^{15}$ Patients with positive findings on physical examination (ie, pain and/or limited range of motion with flexion, adduction, and internal rotation or flexion, abduction, and external rotation) underwent magnetic resonance arthrogram; diagnostic/therapeutic intra-articular anesthetic/corticosteroid injection; and a trial of at least 3 months of nonoperative therapy, including corestrengthening physical therapy. Patients with persistent hip pain despite nonoperative therapy were offered hip arthroscopy.

The senior surgeon began utilizing BMAC in conjunction with hip arthroscopy in December 2016 as a potential method to address concomitant chondral lesions, and all patients who underwent hip arthroscopy gave consent for BMAC preoperatively from this point forward. Thus, the patients who underwent arthroscopic acetabular labral repair with concomitant microfracture to treat fullthickness chondral flaps between June 2014 and November 2016 were compared with similar patients treated with BMAC between December 2016 and April 2020. For the patients who received BMAC, the costs associated with harvesting and application were paid by the Conine Family Fund for Joint Preservation (a philanthropy organization without affiliations to industry); thus, patients' ability to pay played no role in their receiving BMAC. There were no differences in surgical technique (other than microfracture vs BMAC), indications, means of data collection, or rehabilitation between groups. Moreover, the senior surgeon had already completed $>1000$ hip arthroscopy procedures by the time of the first microfracture procedure, thus mitigating any risk of expert bias. ${ }^{27}$

\footnotetext{
*Address correspondence to Michael P. Kucharik, BS, Sports Medicine Center, Massachusetts General Hospital, 175 Cambridge Street, Suite 400 , Boston, MA 02114, USA (email: mikekucharik@gmail.com).

${ }^{\dagger}$ Sports Medicine, Department of Orthopaedic Surgery, Massachusetts General Hospital, Mass General Brigham, Boston, Massachusetts, USA.

${ }^{\ddagger}$ Department of Orthopaedic Surgery, University of Southern California, Los Angeles, California, USA.

§Department of Orthopaedic Surgery, University of Kentucky, Lexington, Kentucky, USA.

"Department of Orthopaedic Surgery, Hospital for Special Surgery, New York, New York, USA.

Final revision submitted August 13, 2021; accepted August 25, 2021.

One or more of the authors has declared the following potential conflict of interest or source of funding: Funding was received from the Conine Family Fund for Joint Preservation for BMAC harvesting and application (S.D.M). S.D.M. has received education payments from Kairos Surgical and honoraria from Allergan. AOSSM checks author disclosures against the Open Payments Database (OPD). AOSSM has not conducted an independent investigation on the OPD and disclaims any liability or responsibility relating thereto.

Ethical approval for this study was obtained from Partners Healthcare (Protocol No. 2013P000722/BWH).
} 

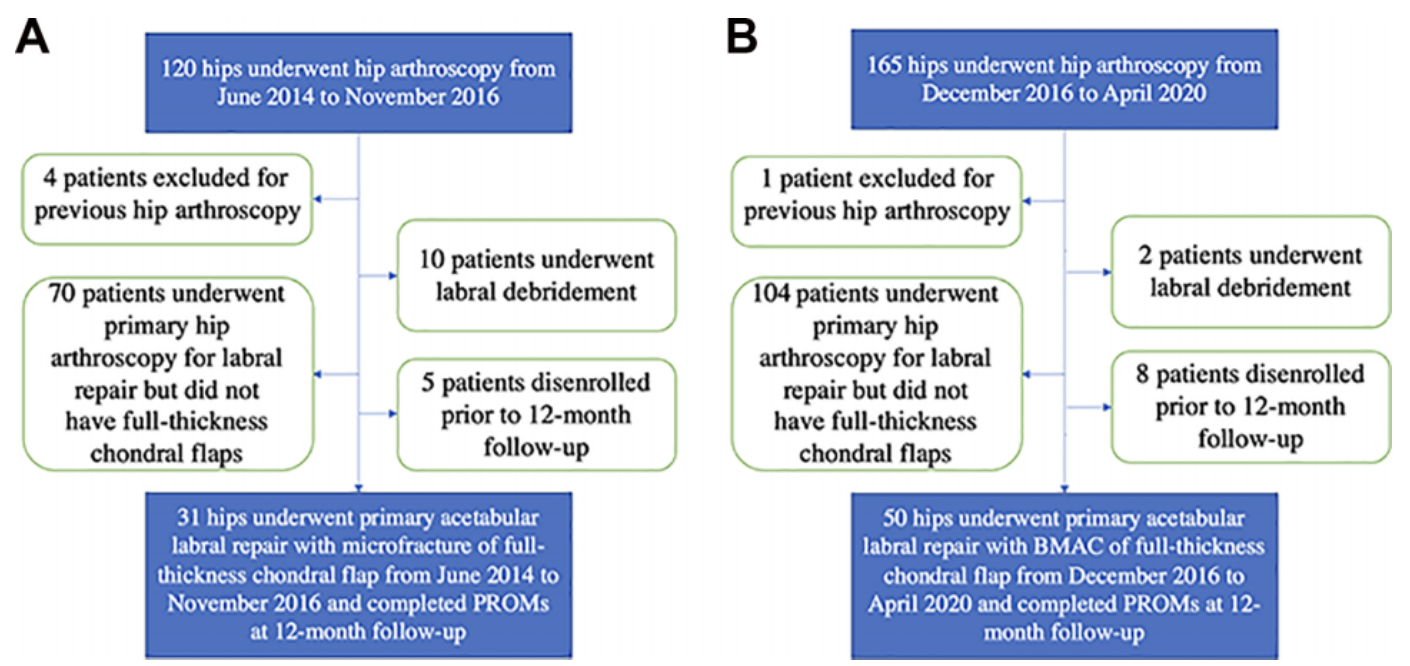

Figure 1. Patient flowcharts for the (A) 31 hips treated with microfracture and (B) 50 hips treated with BMAC included in the study. PROMs, patient-reported outcome measures.

\section{Data Collection}

Patient and descriptive data, including age, sex, laterality, body mass index (BMI), Tönnis grade, and radiographic evidence of FAI, were collected. Intraoperatively, tear size was recorded based on the extent of a clock face converted to degrees. Patients in both cohorts prospectively completed the following PROMs at enrollment before surgery and at 3-, 6-, and 12-month follow-up visits: International Hip Outcome Tool-33 (iHOT-33), Hip Outcome ScoreActivities of Daily Living (HOS-ADL), Hip Outcome Score-Sports Subscale (HOS-Sport), modified Harris Hip Score (mHHS), and visual analog scale (VAS) for pain. Patients were only included for data analysis if they completed PROMs at enrollment and at the 12-month followup. Clinically meaningful outcomes were assessed by calculating the percentage of patients who reached threshold PROM scores for minimal clinically important difference (MCID), patient acceptable symptom state (PASS), and substantial clinical benefit (SCB), as defined by Nwachukwu et al. ${ }^{33}$

\section{Surgical Technique}

The technique of surgical arthroscopic acetabular labral repair using a chondrolabral junction preservation technique with intermittent traction and possible FAI decompression has been described in previous technical notes. ${ }^{6,34,39}$ Once adequate intra-articular visualization of the lesion was established, a blunt obturator probe was used to evaluate the extent of the labral tear, extension of the tear into the chondrolabral junction, and presence of a chondral flap overlying subchondral bone. Notably, acetabular labral repair was performed before microfracture or BMAC harvesting and application. For patients who underwent microfracture, a $60^{\circ}$ microfracture Steadman awl was utilized to carry out perpendicular microfracture into the subchondral plate behind the flap. For patients who underwent BMAC, ${ }^{32}$ processing (Arthrex) and application of BMAC were carried out as described in a previous technical note ${ }^{40}$ For all patients in the BMAC cohort, a BMAC megaclot, composed of $20 \mathrm{~mL}$ of platelet-poor plasma/ platelet-rich plasma and $4 \mathrm{~mL}$ of BMAC along with thrombin for adequate coagulation, was applied into the central compartment of the hip. After arthroscopic acetabular labral repair and microfracture or BMAC, traction was released, and the hip was placed through a full range of motion to ensure restored labral seal as well as excellent stability of the flap.

\section{Postoperative Rehabilitation}

Patients in both cohorts received the same postoperative protocol. Patients were allowed immediate weightbearing as tolerated using a flat-footed gait with crutches for 6 weeks. At 6 weeks postoperatively, patients could start using a stationary bicycle. At 10 weeks, patients were allowed to swim or use an elliptical trainer. At 4 months, strengthening exercises including hamstring curls and short-arc leg press with low weight and high repetitions were encouraged. At 6 months, patients were permitted to resume impact-loading exercises as tolerated.

\section{Statistical Analysis}

Baseline patient factors were compared between the groups using $t$ test or chi-square/Fisher exact test, as appropriate. A 2-tailed paired $t$ test was used to assess differences between preoperative and postoperative scores for the individual groups. Consistent with the repeated-measures data structure, an independent-samples $t$ test was used to compare mean changes in PROMs between the BMAC and microfracture groups. The primary outcome was the mean iHOT-33 score improvement at the 12-month follow-up. All statistics were computed using SPSS statistical software 
TABLE 1

Preoperative and Intraoperative Characteristics for the BMAC and Microfracture Groups ${ }^{a}$

\begin{tabular}{lccc}
\hline & $\begin{array}{c}\text { BMAC } \\
(\mathrm{n}=50 \mathrm{hips})\end{array}$ & $\begin{array}{c}\text { Microfracture } \\
(\mathrm{n}=31 \text { hips })\end{array}$ & $P$ Value \\
\hline Age, $\mathrm{y}$ & $32.8(29.8-35.8)$ & $35.9(31.7-40.1)$ & .242 \\
Sex & & & .949 \\
$\quad$ Male & $31(62.0)$ & $19(61.3)$ & \\
$\quad$ Female & $19(38.0)$ & $12(38.7)$ & \\
Laterality & & & .941 \\
$\quad$ Right & $27(54.0)$ & $17(54.8)$ & \\
Left & $23(46.0)$ & $14(45.2)$ & \\
BMI & $25.4(24.3-26.5)$ & $25.3(23.9-26.7)$ & .913 \\
Tear size, deg & $69.3(63.8-74.8)$ & $67.2(58.2-76.2)$ & .689 \\
Tönnis grade & & & .145 \\
0 & $22(44.0)$ & $7(22.6)$ & \\
1 & $25(50.0)$ & $21(67.7)$ & \\
2 & $3(6.0)$ & $3(9.7)$ & .173 \\
FAI & & & \\
None & $1(2.0)$ & $2(6.4)$ & \\
Pincer & $19(38.0)$ & $14(45.2)$ & \\
Cam & $2(4.0)$ & $4(12.9)$ & \\
Combined & $28(56.0)$ & $11(35.5)$ &
\end{tabular}

${ }^{a}$ Continuous variables are reported as mean $(95 \% \mathrm{CI})$, and categorical variables are reported as $\mathrm{n}(\%)$. BMAC, bone marrow aspirate concentrate; BMI, body mass index; FAI, femoroacetabular impingement.

(version 27.0.0; IBM Corp), and $P<.05$ was considered significant.

\section{RESULTS}

A total of 81 hips ( 81 patients) were included in this study: 50 treated with BMAC and 31 treated with microfracture group (Figure 1). Baseline characteristics were not significantly different between patients in the BMAC and microfracture groups (Table 1). Notably, PROMs for all patients were collected from their 12month follow-up surveys. In terms of disenrollment, $86.2 \%$ and $86.1 \%$ of patients with BMAC and microfracture completed PROMs at the 12-month follow-up, respectively.

In the BMAC cohort, all scores improved significantly from preoperatively to the 12-month follow-up: 41.7 to 75.6 for iHOT-33, 67.6 to 91.0 for HOS-ADL, 41.5 to 72.3 for HOS-Sport, 59.4 to 87.2 for mHHS, and 6.2 to 2.2 for VAS pain $(P<.001$ for all). In the microfracture cohort, significant improvements were seen in the iHOT-33 (48.0 to $65.1 ; P=.001)$, mHHS (70.4 to $78.3 ; P=.028$ ), and VAS pain (4.9 to $3.6 ; P=.036$ ) (Table 2 ).

When comparing results between groups, we found that at baseline, patients who underwent BMAC and microfracture had similar mean iHOT-33 scores (41.7 vs 48.0; $P=.127$ ), and the BMAC group had nonsignificantly higher mean iHOT-33 scores at the 3 -month $(63.0$ vs 55.4 ; $P=.066)$ and 6 -month (70.3 vs $62.4 ; P=.075)$ follow-up visits. However, at the 12-month follow-up, the BMAC
TABLE 2

Comparison of Preoperative and 12-Month Postoperative PROMs Within the BMAC and Microfracture Groups ${ }^{a}$

\begin{tabular}{|c|c|c|c|c|}
\hline & \multicolumn{2}{|l|}{ BMAC } & \multicolumn{2}{|c|}{ Microfracture } \\
\hline & & $P$ & & $P$ \\
\hline & Mean $(95 \%$ CI $)$ & Value & Mean $(95 \%$ CI) & Value \\
\hline iHOT-33 & & $<.001$ & & .001 \\
\hline Preoperative & $41.7(36.7-46.7)$ & & $48.0(41.3-54.8)$ & \\
\hline 12-mo follow-up & $75.6(70.7-80.5)$ & & $65.1(56.7-73.5)$ & \\
\hline HOS-ADL & & $<.001$ & & .275 \\
\hline Preoperative & $67.6(62.2-73.1)$ & & $80.5(74.9-86.2)$ & \\
\hline 12-mo follow-up & $91.0(88.6-93.4)$ & & $83.3(77.0-89.6)$ & \\
\hline HOS-Sport & & $<.001$ & & .568 \\
\hline Preoperative & $41.5(34.5-48.4)$ & & $59.2(51.8-66.5)$ & \\
\hline 12-mo follow-up & $72.3(64.9-79.7)$ & & $62.4(51.4-73.3)$ & \\
\hline mHHS & & $<.001$ & & .028 \\
\hline Preoperative & $59.4(55.2-63.6)$ & & $70.4(66.2-74.6)$ & \\
\hline 12-mo follow-up & $87.2(84.2-90.1)$ & & $78.3(72.6-84.0)$ & \\
\hline VAS pain & & $<.001$ & & .036 \\
\hline Preoperative & $6.2(5.4-6.8)$ & & $4.9(4.0-5.9)$ & \\
\hline 12-mo follow-up & $2.2(1.5-2.8)$ & & $3.6(2.7-4.6)$ & \\
\hline
\end{tabular}

${ }^{a}$ Bolded $P$ values indicate a statistically significant improvement from preoperatively to follow-up $(P<.05)$. BMAC, bone marrow aspirate concentrate; HOS-ADL, Hip Outcome ScoreActivities of Daily Living; HOS-Sport, Hip Outcome Score-Sports Subscale; iHOT-33, International Hip Outcome Tool-33; mHHS, modified Harris Hip Score; PROMs, patient-reported outcome measures; VAS, visual analog scale.

cohort had significantly higher mean iHOT-33 scores compared with the microfracture cohort ( 75.6 vs $65.1 ; P=.025)$ (Table 3).

In terms of improvement in mean iHOT-33 scores, the BMAC cohort reported significantly greater improvements from baseline at the 3 -month $(22.0$ vs $7.9 ; P=.004)$, 6 -month ( 27.7 vs $13.8 ; P=.010)$, and 12 -month (33.9 vs $15.2 ; P<.001$ ) follow-up visits (Table 4 ).

For HOS-ADL, despite significantly lower baseline scores for the BMAC cohort (68.2 vs $79.1 ; P=.007$ ), the BMAC cohort still outperformed the microfracture cohort at the 12 -month follow-up (91.0 vs $83.3 ; P=.032)$. Similarly, the BMAC cohort reported significantly lower mean mHHS values at baseline ( 59.4 vs $70.6 ; P=.001)$ but significantly higher mean mHHS values at the 12 -month follow-up (87.2 vs 78.3; $P=.003$ ). Moreover, the BMAC cohort reported lower mean VAS scores at the 12-month follow-up ( 2.2 vs $3.6 ; P=.012)$, despite higher mean pain scores preoperatively $(6.1$ vs $5.0 ; P=.072)$.

In terms of clinically meaningful outcomes, $77.6 \%$ and $50.0 \%$ of hips treated with BMAC application and microfracture achieved 12-month improvements in iHOT-33 scores that reached the MCID threshold, respectively $(P=.013)$. Otherwise, the only significant differences between groups for PASS and SCB were the mHHS: BMAC, $58.0 \%$ versus microfracture, $25.0 \%(P=.008)$ for PASS and BMAC, $56.0 \%$ versus microfracture, $25.0 \%(P=.012)$ for SCB (Table 5). 
TABLE 3

Comparison of PROM Scores Over Time Between the BMAC and Microfracture Groups ${ }^{a}$

\begin{tabular}{lccc}
\hline & BMAC & Microfracture & $P$ Value \\
\hline Enrollment & $\mathrm{n}=50$ & $\mathrm{n}=31$ & \\
iHOT-33 & $41.7(36.9-46.5)$ & $48.0(41.3-54.8)$ & .127 \\
HOS-ADL & $68.2(62.8-73.6)$ & $79.1(73.6-84.6)$ & $\mathbf{. 0 0 7}$ \\
HOS-Sport & $42.4(35.6-49.1)$ & $56.4(48.8-64.1)$ & $\mathbf{. 0 1 0}$ \\
mHHS & $59.4(55.2-63.6)$ & $70.6(66.3-75.0)$ & $\mathbf{. 0 0 1}$ \\
VAS pain & $6.1(5.4-6.8)$ & $5.0(4.1-6.0)$ & .072 \\
3-mo follow-up & $\mathrm{n}=44$ & $\mathrm{n}=23$ & \\
iHOT-33 & $63.0(58.6-67.3)$ & $55.4(48.4-62.5)$ & .066 \\
HOS-ADL & $82.2(78.4-85.9)$ & $74.6(67.8-81.4)$ & $\mathbf{. 0 4 2}$ \\
HOS-Sport & $43.6(35.0-52.3)$ & $28.5(19.2-37.8)$ & $\mathbf{. 0 3 2}$ \\
mHHS & $77.6(74.1-81.2)$ & $72.6(67.2-78.1)$ & .127 \\
VAS pain & $2.7(2.0-3.3)$ & $3.0(2.1-3.9)$ & .526 \\
6-mo follow-up & $\mathrm{n}=46$ & $\mathrm{n}=22$ & \\
iHOT-33 & $70.3(65.6-75.0)$ & $62.4(54.9-70.0)$ & .075 \\
HOS-ADL & $87.4(84.0-90.7)$ & $81.5(75.9-87.1)$ & .067 \\
HOS-Sport & $63.6(55.6-71.6)$ & $51.0(40.7-61.2)$ & .071 \\
mHHS & $81.4(77.8-85.1)$ & $76.8(71.0-82.6)$ & .177 \\
VAS pain & $2.4(1.8-3.1)$ & $3.0(2.0-3.9)$ & .377 \\
12-mo follow-up & $\mathrm{n}=50$ & $\mathrm{n}=31$ & \\
iHOT-33 & $75.6(70.7-80.5)$ & $65.1(56.7-73.5)$ & $\mathbf{. 0 2 5}$ \\
HOS-ADL & $91.0(88.6-93.4)$ & $83.3(77.0-89.6)$ & $\mathbf{. 0 3 2}$ \\
HOS-Sport & $72.3(64.9-79.7)$ & $62.4(51.4-73.3)$ & .132 \\
mHHS & $87.2(84.2-90.1)$ & $78.3(72.6-84.0)$ & $\mathbf{. 0 0 3}$ \\
VAS pain & $2.2(1.5-2.8)$ & $3.6(2.7-4.6)$ & $\mathbf{. 0 1 2}$ \\
\hline
\end{tabular}

${ }^{a}$ Data are reported as mean $(95 \% \mathrm{CI})$. Bolded $P$ values indicate a statistically significant difference between groups $(P<.05)$. BMAC, bone marrow aspirate concentrate; HOS-ADL, Hip Outcome Score-Activities of Daily Living; HOS-Sport, Hip Outcome Score-Sports Subscale; iHOT-33, International Hip Outcome Tool-33; mHHS, modified Harris Hip Score; PROM, patientreported outcome measure; VAS, visual analog scale.

\section{DISCUSSION}

In the current study, both BMAC and microfracture cohorts experienced significant improvements in mean iHOT-33 score from baseline to the 12-month follow-up; however, the BMAC cohort outperformed the microfracture cohort at 12 months in terms of raw score and improvement at statistically significant levels. Moreover, the difference between groups at the 12-month follow-up was clinically meaningful, as a higher percentage of patients in the BMAC cohort achieved improvements that met the MCID threshold for iHOT-33 scores. ${ }^{33}$

That BMAC and microfracture cohorts experienced significant improvements from baseline is not surprising, as previous studies have reported positive results for other techniques that can preserve chondral flaps that contain up to $90 \%$ viable chondrocytes ${ }^{17,29,36,43}$ As described by Levinson et al, ${ }^{24}$ the difficulty with preservation of the flap has been more so related to maintaining the integrity of the ECM around the chondrocytes. Once the chondral flap ruptures from the underlying subchondral bone, the ECM becomes disturbed, and remaining chondrocytes are unable to deposit new ECM regardless of their proximity to adjacent bone. ${ }^{24,43}$ This phenomenon served as the impetus for
TABLE 4

Comparison of Changes From Baseline in PROM Scores Between the BMAC and Microfracture Groups ${ }^{a}$

\begin{tabular}{lccr}
\hline & & & \\
& BMAC & Microfracture & $\begin{array}{c}P \\
\text { Value }\end{array}$ \\
\hline 3-mo follow-up & $\mathrm{n}=44$ & $\mathrm{n}=23$ & \\
$\Delta$ iHOT-33 & $22.0(16.6$ to 27.4$)$ & $7.9(0.1$ to 15.8$)$ & $\mathbf{. 0 0 4}$ \\
$\Delta$ HOS-ADL & $15.3(10.2$ to 20.3$)$ & $-3.5(-12.6$ to 5.5$)$ & $<.001$ \\
$\Delta$ HOS-Sport & $4.2(-4.1$ to 12.4$)$ & $-25.0(-35.7$ to -14.2$)$ & $<.001$ \\
$\Delta$ mHHS & $18.1(12.9$ to 23.2$)$ & $1.6(-5.5$ to 8.8$)$ & $<.001$ \\
$\Delta$ VAS & $-3.4(-4.2$ to -2.5$)$ & $-1.9(-3.0$ to -0.8$)$ & $\mathbf{. 0 4 0}$ \\
6-mo follow-up & $\mathrm{n}=46$ & $\mathrm{n}=22$ & \\
$\Delta$ iHOT-33 & $27.7(21.8$ to 33.5$)$ & $13.8(5.3$ to 22.2$)$ & $\mathbf{. 0 1 0}$ \\
$\Delta$ HOS-ADL & $18.4(12.6$ to 24.1$)$ & $2.9(-2.3$ to 8.2$)$ & $<.001$ \\
$\Delta$ HOS-Sport & $19.4(9.6$ to 29.2$)$ & $-4.9(-15.4$ to 5.5$)$ & $\mathbf{. 0 0 4}$ \\
$\Delta$ mHHS & $20.4(14.9$ to 26.0$)$ & $6.4(-0.2$ to 12.8$)$ & $\mathbf{. 0 0 4}$ \\
$\Delta$ VAS & $-3.5(-4.3$ to -2.6$)$ & $-1.7(-2.7$ to -0.6$)$ & $\mathbf{. 0 1 8}$ \\
12-mo follow-up & $\mathrm{n}=50$ & $\mathrm{n}=31$ & \\
$\Delta$ iHOT-33 & $33.9(27.8$ to 40.0$)$ & $15.2(6.9$ to 23.5$)$ & $<.001$ \\
$\Delta$ HOS-ADL & $23.4(18.0$ to 28.8$)$ & $2.8(-2.1$ to 7.7$)$ & $<.001$ \\
$\Delta$ HOS-Sport & $30.8(21.8$ to 39.8$)$ & $3.1(-7.6$ to 13.8$)$ & $<.001$ \\
$\Delta$ mHHS & $27.8(22.5$ to 33.0$)$ & $7.8(1.2$ to 14.4$)$ & $<.001$ \\
$\Delta$ VAS & $-4.0(-4.8$ to -3.1$)$ & $-1.3(-2.1$ to -0.4$)$ & $<.001$ \\
\hline
\end{tabular}

${ }^{a}$ Data are reported as mean $(95 \% \mathrm{CI})$. Bolded $P$ values indicate a statistically significant difference between groups $(P<.05)$. BMAC, bone marrow aspirate concentrate; HOS-ADL, Hip Outcome Score-Activities of Daily Living; HOS-Sport, Hip Outcome Score-Sports Subscale; iHOT-33, International Hip Outcome Tool-33; mHHS, modified Harris Hip Score; PROM, patientreported outcome measure; VAS, visual analog scale for pain.

the evolution of surgical techniques to replace functional ECM to act as a bridge between viable chondrocytes and exposed subchondral bone. ${ }^{12,26,38,42}$ Thus, techniques such as BMAC, microfracture, or a fibrin adhesive would be ideal strategies to reduce the chondral flap and restore its native anatomy while maintaining the survival of viable chondrocytes.

The current study adds to the growing body of literature regarding treatment strategies for full-thickness chondral flaps. Haefeli et $\mathrm{al}^{16}$ found that subchondral drilling under the chondral flap decreased the rate of conversion to THA when compared with simple debridement of the chondral flap. Similarly, Hevesi et al ${ }^{19}$ compared microfracture and debridement and reported mHHS, HOS-Sport, and VAS improvements that were similar to those of the microfracture cohort in our study. However, Hevesi et al $^{19}$ did not find a statistically significant difference between groups in terms of functional outcomes or long-term survival with a follow-up of 5 years. Similar to the current study, Ivone et $\mathrm{al}^{20}$ found that the microfragmented autologous adipose tissue transplantation technique group reported a final mHHS that outperformed the microfracture cohort by $>1$ MCID threshold. Tahoun et $\mathrm{a}^{41}$ utilized a chitosanbased scaffold (BST-CarGel) alongside microfracture and found that $>90 \%$ of patients demonstrated complete restoration of the cartilage defect on magnetic resonance imaging scans with specific cartilage sequences. Lastly, Tzaveas and Villar ${ }^{42}$ utilized a fibrin adhesive without 
TABLE 5

Comparison of Clinically Meaningful Outcomes Between the BMAC and Microfracture Groups ${ }^{a}$

\begin{tabular}{lrrr}
\hline & BMAC & Microfracture & $P$ Value \\
\hline MCID & & & \\
iHOT-33 & $38(77.6)$ & $14(50.0)$ & .013 \\
HOS-ADL & $33(66.0)$ & $7(25.0)$ & $<.001$ \\
HOS-Sport & $37(77.1)$ & $10(35.7)$ & $\mathbf{< . 0 0 1}$ \\
mHHS & $42(84.0)$ & $13(48.1)$ & $\mathbf{< . 0 0 1}$ \\
PASS & & & \\
iHOT-33 & $32(66.7)$ & $14(56.0)$ & .206 \\
HOS-ADL & $33(67.3)$ & $14(56.0)$ & .183 \\
HOS-Sport & $29(60.4)$ & $12(48.0)$ & .310 \\
mHHS & $29(58.0)$ & $6(25.0)$ & .008 \\
SCB & & & .243 \\
iHOT-33 & $27(56.3)$ & $10(40.0)$ & .450 \\
HOS-ADL & $33(67.3)$ & $14(56.0)$ & .871 \\
HOS-Sport & $24(50.0)$ & $12(48.0)$ & .012 \\
mHHS & $28(56.0)$ & $6(25.0)$ & \\
\hline
\end{tabular}

${ }^{a}$ Data are reported as No. of patients (\%). Bolded $P$ values indicate a statistically significant difference between groups $(P<.05)$. BMAC, bone marrow aspirate concentrate; HOS-ADL, Hip Outcome Score-Activities of Daily Living; HOS-Sport, Hip Outcome Score-Sports Subscale; iHOT-33, International Hip Outcome Tool-33; MCID, minimal clinically important differences; mHHS, modified Harris Hip Score; PASS, patient acceptable symptom state; SCB, substantial clinical benefit.

microfracture and reported significantly improved clinical outcomes when compared with baseline scores. While microfracture in the aforementioned studies was reported as safe, some studies have found a greater long-term progression to THA and revision arthroscopy in patients undergoing microfracture of the hip than in a control cohort. $^{9,11,25}$ Thus, our study introduces an alternative technique for preservation of the chondral flap with better short-term clinical outcomes when compared with a standard treatment that has been associated with a long-term risk of conversion to THA.

While this study has several strengths, including its large sample of patients undergoing hip arthroscopy with BMAC treatment for full-thickness chondral flaps with prospectively collected outcome measures and its utilization of a similar control cohort for comparison, it is not without limitations. First, patients who received BMAC or microfracture did not undergo postoperative magnetic resonance imaging, which could have been used to assess cartilage healing after surgical intervention. Second, while the surgeon was highly experienced at the time the first patient underwent microfracture and there were no differences in surgical technique or rehabilitation between cohorts, unobserved confounders that occurred over time may have contributed to the results. Third, as is the case with survey studies, loss to follow-up and unanswered questionnaires serve as a potential source of bias. Importantly, this source of bias should not differ between groups, as methods of enrollment and survey collection have not changed since the first patient who underwent microfracture. Fourth, while patients in the BMAC cohort experienced greater PROM improvements than did those who underwent microfracture, it is important to note that patients who underwent microfracture had higher scores preoperatively, albeit at a statistically insignificant level. While this may have potentially affected the percentage of patients who reached MCID, the BMAC cohort still reported significantly greater iHOT-33 scores at the 12-month follow-up, despite starting at lower baseline scores. Fifth, BMAC harvesting and application during hip arthroscopy is a relatively novel procedure and, thus, is unavailable to many patients because of financial or logistical limitations. Sixth, because of the novelty of BMAC for the treatment of full-thickness chondral flaps, long-term follow-up is needed to determine sustainable outcomes and progression to THA. Notably, the results of the current study are preliminary and merely suggest an alternative treatment option for full-thickness chondral flaps, as the microfracture cohort still experienced significant improvements, which is consistent with results in the current literature.

\section{CONCLUSION}

Patients with full-thickness chondral flaps at the time of hip arthroscopy experienced greater improvements in functional outcome scores at the 12-month follow-up when treated with BMAC as opposed to microfracture. These findings are preliminary, and future, high-level studies examining the long-term utility of BMAC for the treatment of chondral flaps are needed.

\section{REFERENCES}

1. Abrams GD, Frank RM, Fortier LA, Cole BJ. Platelet-rich plasma for articular cartilage repair. Sports Med Arthrosc Rev. 2013;21(4): 213-219.

2. Amanatullah DF, Antkowiak T, Pillay K, et al. Femoroacetabular impingement: current concepts in diagnosis and treatment. Orthopedics. 2015;38(3):185-199.

3. Anderson LA, Peters CL, Park BB, et al. Acetabular cartilage delamination in femoroacetabular impingement: risk factors and magnetic resonance imaging diagnosis. J Bone Joint Surg Am. 2009;91(2): 305-313.

4. Burkart A, Imhoff AB. Therapy of cartilage damage-more current than ever. Article in German. Unfallchirurg. 2001;104(9):797.

5. Chahla J, Dean CS, Moatshe G, et al. Concentrated bone marrow aspirate for the treatment of chondral injuries and osteoarthritis of the knee: a systematic review of outcomes. Orthop J Sports Med. 2016; 4(1):2325967115625481.

6. Conaway WK, Martin SD. Puncture capsulotomy during hip arthroscopy for femoroacetabular impingement: preserving anatomy and biomechanics. Arthrosc Tech. 2017;6(6):e2265-e2269.

7. Cotter EJ, Wang KC, Yanke AB, Chubinskaya S. Bone marrow aspirate concentrate for cartilage defects of the knee: from bench to bedside evidence. Cartilage. 2018;9(2):161-170.

8. Crawford K, Philippon MJ, Sekiya JK, Rodkey WG, Steadman JR. Microfracture of the hip in athletes. Clin Sports Med. 2006;25(2): 327-335, x. 
9. Dallich AA, Rath E, Atzmon R, et al. Chondral lesions in the hip: a review of relevant anatomy, imaging and treatment modalities. $J$ Hip Preserv Surg. 2019;6(1):3-15.

10. Domb BG, El Bitar YF, Lindner D, Jackson TJ, Stake CE. Arthroscopic hip surgery with a microfracture procedure of the hip: clinical outcomes with two-year follow-up. Hip Int. 2014;24(5):448-456.

11. Domb BG, Gupta A, Dunne KF, et al. Microfracture in the hip: results of a matched-cohort controlled study with 2-year follow-up. Am J Sports Med. 2015;43(8):1865-1874.

12. Fontana A, Bistolfi A, Crova M, Rosso F, Massazza G. Arthroscopic treatment of hip chondral defects: autologous chondrocyte transplantation versus simple debridement-a pilot study. Arthroscopy. 2012; 28(3):322-329.

13. Fontana A, Mancini D, Gironi A, Acerbi A. Hip osteochondral lesions: arthroscopic evaluation. Hip Int. 2016;26(suppl 1):17-22.

14. Green CJ, Beck A, Wood D, Zheng MH. The biology and clinical evidence of microfracture in hip preservation surgery. $J$ Hip Preserv Surg. 2016;3(2):108-123.

15. Griffin DR, Dickenson EJ, O'Donnell J, et al. The Warwick Agreement on femoroacetabular impingement syndrome (FAl syndrome): an international consensus statement. Br J Sports Med. 2016;50(19): 1169-1176.

16. Haefeli PC, Tannast M, Beck M, Siebenrock KA, Buchler L. Subchondral drilling for chondral flaps reduces the risk of total hip arthroplasty in femoroacetabular impingement surgery at minimum five years follow-up. Hip Int. 2019;29(2):191-197.

17. Hariri S, Truntzer J, Smith RL, Safran MR. Biochemical and cellular assessment of acetabular chondral flaps identified during hip arthroscopy. Arthroscopy. 2015;31(6):1077-1083.

18. Hatakeyama A, Utsunomiya $\mathrm{H}$, Nishikino $\mathrm{S}$, et al. Predictors of poor clinical outcome after arthroscopic labral preservation, capsular plication, and cam osteoplasty in the setting of borderline hip dysplasia. Am J Sports Med. 2018;46(1):135-143.

19. Hevesi M, Bernard C, Hartigan DE, et al. Is microfracture necessary? Acetabular chondrolabral debridement/abrasion demonstrates similar outcomes and survival to microfracture in hip arthroscopy: a multicenter analysis. Am J Sports Med. 2019;47(7):1670-1678.

20. Ivone A, Fioruzzi A, Jannelli E, et al. Micro-fragmented adipose tissue transplantation (MATT) for the treatment of acetabular delamination: a two years follow up comparison study with microfractures. Acta Biomed. 2019;90(12-S):69-75.

21. Jannelli E, Parafioriti A, Acerbi A, et al. Acetabular delamination: epidemiology, histological features, and treatment. Cartilage. 2019;10(3): 314-320.

22. Johnston TL, Schenker ML, Briggs KK, Philippon MJ. Relationship between offset angle alpha and hip chondral injury in femoroacetabular impingement. Arthroscopy. 2008;24(6):669-675.

23. Kraeutler MJ, Goodrich JA, Fioravanti MJ, Garabekyan T, Mei-Dan O. The "outside-in" lesion of hip impingement and the "inside-out" lesion of hip dysplasia: two distinct patterns of acetabular chondral injury. Am J Sports Med. 2019;47(12):2978-2984.

24. Levinson C, Naal FD, Salzmann GM, Zenobi-Wong M, Leunig M. Is there a scientific rationale for the refixation of delaminated chondral flaps in femoroacetabular impingement? A laboratory study. Clin Orthop Relat Res. 2020;478(4):854-867.

25. Lodhia P, Gui C, Chandrasekaran S, et al. Microfracture in the hip: a matched-control study with average 3-year follow-up. J Hip Preserv Surg. 2015;2(4):417-427.
26. Mancini D, Fontana A. Five-year results of arthroscopic techniques for the treatment of acetabular chondral lesions in femoroacetabular impingement. Int Orthop. 2014;38(10):2057-2064.

27. Mehta N, Chamberlin P, Marx RG, et al. Defining the learning curve for hip arthroscopy: a threshold analysis of the volume-outcomes relationship. Am J Sports Med. 2018;46(6):1284-1293.

28. Menge TJ, Briggs KK, Dornan GJ, McNamara SC, Philippon MJ. Survivorship and outcomes 10 years following hip arthroscopy for femoroacetabular impingement: labral debridement compared with labral repair. J Bone Joint Surg Am. 2017;99(12):997-1004.

29. Meulenkamp B, Gravel D, Beaule PE. Viability assessment of the chondral flap in patients with cam-type femoroacetabular impingement: a preliminary report. Can J Surg. 2014;57(1):44-48.

30. Mygind-Klavsen B, Gronbech Nielsen T, Maagaard N, et al. Danish Hip Arthroscopy Registry: an epidemiologic and perioperative description of the first 2000 procedures. J Hip Preserv Surg. 2016; 3(2):138-145.

31. Nasser R, Domb B. Hip arthroscopy for femoroacetabular impingement. EFORT Open Rev. 2018;3(4):121-129.

32. Nazal MR, McCarthy MBR, Mazzocca AD, Martin SD. Connective tissue progenitor analysis of bone marrow aspirate concentrate harvested from the body of the ilium during arthroscopic acetabular labral repair. Arthroscopy. 2020;36(5):1311-1320.

33. Nwachukwu BU, Beck EC, Kunze KN, et al. Defining the clinically meaningful outcomes for arthroscopic treatment of femoroacetabular impingement syndrome at minimum 5-year follow-up. Am J Sports Med. 2020;48(4):901-907.

34. Nwachukwu BU, McCormick F, Martin SD. Arthroscopic technique for chondrolabral capsular preservation during labral repair and acetabular osteoplasty. Arthrosc Tech. 2013;2(3):e213-e216.

35. Pfirrmann CW, Mengiardi B, Dora C, et al. Cam and pincer femoroacetabular impingement: characteristic MR arthrographic findings in 50 patients. Radiology. 2006;240(3):778-785.

36. Rodriguez-Fontan F, Payne KA, Chahla J, et al. Viability and tissue quality of cartilage flaps from patients with femoroacetabular hip impingement: a matched-control comparison. Orthop J Sports Med. 2017;5(8):2325967117723608.

37. Sansone M, Ahlden M, Jonasson $P$, et al. A Swedish hip arthroscopy registry: demographics and development. Knee Surg Sports Traumatol Arthrosc. 2014;22(4):774-780.

38. Sekiya JK, Martin RL, Lesniak BP. Arthroscopic repair of delaminated acetabular articular cartilage in femoroacetabular impingement. Orthopedics. 2009;32(9).

39. Skelley NW, Conaway WK, Martin SD. "In-round" labral repair after acetabular recession using intermittent traction. Arthrosc Tech. 2017; 6(5):e1807-e1813.

40. Stelzer JW, Martin SD. Use of bone marrow aspirate concentrate with acetabular labral repair for the management of chondrolabral junction breakdown. Arthrosc Tech. 2018;7(10):e981-e987.

41. Tahoun M, Shehata TA, Ormazabal I, et al. Results of arthroscopic treatment of chondral delamination in femoroacetabular impingement with bone marrow stimulation and BST-CarGel ${ }^{\circledR}$. SICOT-J. 2017; 3(51).

42. Tzaveas AP, Villar RN. Arthroscopic repair of acetabular chondral delamination with fibrin adhesive. Hip Int. 2010;20(1):115-119.

43. Wright VJ, McCrum CL, Li H, Tranovich MJ, Huard J. Significant chondrocyte viability is present in acetabular chondral flaps associated with femoroacetabular impingement. Am J Sports Med. 2018; 46(1):149-152. 